\title{
Level Control of Quadruple Tank Process using Laguerre Functions based Model Predictive Control Algorithm
}

\author{
Kamel Menighed ${ }^{(1)^{*}}$, Issam Chekakta ${ }^{(2)}$ \\ ${ }^{(1)}$ Dept. of Petrochemicals and process engineering,University of 20 août 1955,Skikda, Algeria \\ (2) Ferhat ABBAS University-DAC HR Laboratory, Sétif1, Algeria \\ k.menighed@univ-skikda.dz
}

\begin{abstract}
This paper aims to present a model predictive controller based on discrete state-space modeling, where the future control trajectory is approximated by a set of discrete-time Laguerre functions instead of shift forward operators. The benefit of using these orthonormal Laguerre functions is that they have fewer parameters to adjust in the optimization problem and the computation load is significantly lower than the standard predictive control. The effectiveness of this controller is illustrated through the quadruple tank process, which is a highly interacted, multivariable and constrained system.
\end{abstract}

Keywords: Discrete-linear state-space, Model Predictive Control (MPC), Laguerre functions, Optimization.

\section{INTRODUCTION}

In last few decade, many control strategies for multivariable systems have been in high demand and need much attention in the process industry, where the existence of interactions known as coupling, hard constraints and time delays is inevitable which may lead to instability and insufficient performances, where the classical strategies faced many difficulties such as PID control,[10], [12], [14], [15], [16]. To overcome these complex behaviors many researchers looked forward in order to find the new control solutions such as [18], the procedure to design the internal model control for multivariable systems is introduced in [2]. Stein [11] used the linear Quadratic Gaussian methodology to design feedback compensators. Panagopoulos et al [9] proposed a PID controller based on optimization with constraints. In [4] a predictive PID controllers for SISO systems is proposed.

The Generalized Model Predictive Control, one of the early basic strategies of Model Predictive Control, was introduced by Clarke in 1987 [1] and received considerable attention from both academia and industry communities.

Despite the increasing demands on this strategy for industrial applications, there were some limitations due to the computation time needed to implement such controller to satisfy the control performances. Morari et al [8] introduced model predictive control using state-space models which facilitates the extension to more complex cases such as multivariable systems with time delay, systems with uncertainties or stochastic systems.

Recalling that the MPC strategy is based on an on-line optimization problem and uses a process model to predict the effect of the control sequence on the behavior of the plant. From the control point of view, it is well known that MPC approach uses the current states of the system, the current output measurements and the reference trajectory to calculate the future control signal by optimizing an objective function subject to a variety of constraints. Since one of the major issues of the conventional MPC is the number of forward shift operator needed in the control horizon to obtain the best control performance, which makes the computational load very heavy. In order to reduce the computational burden in the rolling optimization with a sufficiently large prediction horizon, the orthonormal Laguerre functions are used to approximate the predicted control trajectory by using a fewer number of parameters and obtain the same performance as the conventional MPC control approach. It is worth to emphasize that the MPC/Laguerre approach is becoming an important topic in modern control theory and practice.

The paper is organized as follows. In section.2, we give the system model 
representation forQuadruple Tank process, also we recall some preliminaries about the Laguerre functions. Section.3 describes the control trajectory based on Laguerre functions and the controller design. Simulation results are given in section.4 to illustrate the effectiveness of the proposed control scheme. Finally, a conclusion and future work are presented in Section.5.

\section{PROBLEM STATEMENT AND PRELIMINARIES}

\section{Mathematical Model of the QTP}

The Quadruple Tank Process $(Q T P)$ described in [3] is a multi-tank version of [17] which consists of four interconnected water tanks with two pumps and fixed valve positions (see Fig.1). Pump $A$ pours water into the bottom left tank (Tank 1) and the upper right tank (Tank 4), while pump $B$ pours water into the bottom right tank (Tank 2) and the upper left tank (Tank 3), and water from the two upper tanks flows into the two lower tanks, changing the positions of the valves changes the system behavior changes to either minimum or nonminimum phase behavior, which is an interesting feature of this process.

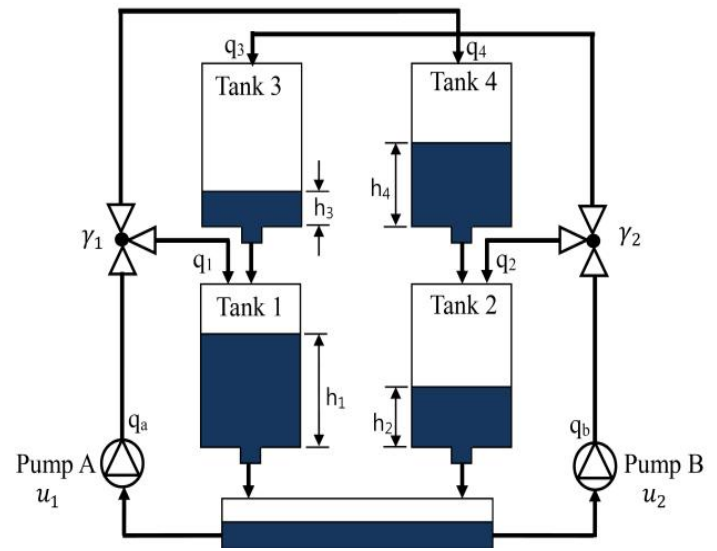

Fig. 1. A schematic representation of the $Q T P$

The goal is to control the water levels $h_{1}$ and $h_{2}$ of tanks 1 and 2 using the pump command voltages $v_{1}$ and $v_{2}$.

Let $\quad x=\left[x_{1} x_{2} x_{3} x_{4}\right]^{T}=\left[h_{1} h_{2} h_{3} h_{4}\right]^{T}$ and $u=\left[u_{1} u_{2}\right]^{T}=\left[v_{1} v_{2}\right]^{T}$ with $h_{i}$ and $v_{j}$ are the variations of the levels and the pumps voltages, respectively.

In order to obtain the mathematical model of the QTP Bernoulli's law and mass balance law are used for each tank. $\frac{d(\rho v)}{d t}=\rho q_{\text {in }}-\rho q_{\text {out }}, A_{i} \frac{d h_{i}}{d t}=q_{\text {in }}-q_{\text {out }}(1)$

where $\rho$ is density, $A_{i}$ is the cross-sectional area of the tank, $h_{i}$ the tank water level, and $q_{\left\{i_{i}\right\}}, q_{\left\{\text {out }_{i}\right\}}$ are the inflow and the outflow of the tank, respectively. Due to the structure of this process, the inflow and the outflow of the tank can be written as follows:

$$
\begin{gathered}
q_{i n_{1}}=k_{1} u_{1}, \quad q_{i n_{3}}=k_{2} u_{2}\left(1-\gamma_{2}\right) \\
q_{i n_{2}}=k_{2} u_{2}, \quad q_{i n_{4}}=k_{1} u_{1}\left(1-\gamma_{1}\right) \\
q_{\text {out }_{i}}=a_{i} \sqrt{2 g h_{i}}(2)
\end{gathered}
$$

where $k_{1}, k_{2}$ are pump constants, $\gamma_{1}, \gamma_{2}$ are the value ratios of the valve positions, $a_{i}$ is cross sectional area of the outlet pipes and $g$ is gravity. The overall nonlinear model is given by

$$
\begin{gathered}
A_{1} \frac{d h_{1}}{d t}=-a_{1} \sqrt{2 g h_{1}}+a_{3} \sqrt{2 g h_{3}}+\gamma_{1} k_{1} u_{1} \\
A_{2} \frac{d h_{2}}{d t}=-a_{2} \sqrt{2 g h_{2}}+a_{4} \sqrt{2 g h_{4}}+\gamma_{2} k_{2} u_{2} \\
A_{3} \frac{d h_{3}}{d t}=-a_{3} \sqrt{2 g h_{3}}+\left(1-\gamma_{2}\right) k_{2} u_{2} \\
A_{4} \frac{d h_{4}}{d t}=-a_{4} \sqrt{2 g h_{4}}+\left(1-\gamma_{1}\right) k_{1} u_{1}
\end{gathered}
$$

To design the controller the system should be linearized around operating points using Taylor expansion. Due to the space limitation, the details of the linearization have been omitted. Therefore, the linearized model is given by

$$
\begin{aligned}
& \dot{x}(t)=\left[\begin{array}{cccc}
-\frac{1}{T_{1}} & 0 & \frac{A_{3}}{A_{1} T_{3}} & 0 \\
0 & -\frac{1}{T_{2}} & 0 & \frac{A_{4}}{A_{2} T_{4}} \\
0 & 0 & -\frac{1}{T_{3}} & 0 \\
0 & 0 & 0 & -\frac{1}{T_{4}}
\end{array}\right] x(t) \\
& +\left[\begin{array}{cc}
\frac{\gamma_{1} k_{1}}{A_{1}} & 0 \\
0 & \frac{\gamma_{2} k_{2}}{A_{2}} \\
0 & \frac{\left(1-\gamma_{2}\right) k_{2}}{A_{3}} \\
\frac{\left(1-\gamma_{1}\right) k_{1}}{A_{4}} & 0
\end{array}\right] u(t) \\
& y(t)=\left[\begin{array}{llll}
1 & 0 & 0 & 0 \\
0 & 1 & 0 & 0
\end{array}\right] x(t)(4)
\end{aligned}
$$


with $T_{i}=\frac{A_{i}}{a_{i}} \sqrt{\frac{2 h_{i 0}}{g}}$.

As mentioned before, by changing the values of $\gamma_{1}, \gamma_{2}$, the system behavior changes to minimum or non-minimum phase behavior as shown in Table 1

Table1 Valve settings

\begin{tabular}{|c|l|l|}
\hline Valve Values & Process & Zero Location \\
\hline $\begin{array}{l}1<\gamma_{1}+\gamma_{2} \\
<2\end{array}$ & $\begin{array}{l}\text { Minimum- } \\
\text { phase }\end{array}$ & $\begin{array}{l}\text { Zero in the left } \\
\text { half plan }\end{array}$ \\
\hline $\begin{array}{l}0<\gamma_{1}+\gamma_{2} \\
<1\end{array}$ & $\begin{array}{l}\text { Non -minimum } \\
\text { phase }\end{array}$ & $\begin{array}{l}\text { Zero in the } \\
\text { right half plan }\end{array}$ \\
\hline$\gamma_{1}+\gamma_{2}=1$ & & $\begin{array}{l}\text { Zero is at the } \\
\text { origin }\end{array}$ \\
\hline
\end{tabular}

The stationary operating conditions, $x_{0}$ and $u_{0}$, are given in Table 2

Table 2 Stationary values

\begin{tabular}{|c|c|c|}
\hline Parameters & $\begin{array}{l}\text { Non-minimum } \\
\text { phase }\end{array}$ & $\begin{array}{l}\text { minimum } \\
\text { phase }\end{array}$ \\
\hline$h_{1_{0}}[\mathrm{~cm}]$ & 8.24 & 12.26 \\
\hline$h_{20}[\mathrm{~cm}]$ & 19.02 & 12.78 \\
\hline$h_{3_{0}}[\mathrm{~cm}]$ & 4.32 & 1.63 \\
\hline$h_{4_{0}}[\mathrm{~cm}]$ & 8.81 & 1.41 \\
\hline$u_{1_{0}}[\mathrm{v}]$ & 3 & 3 \\
\hline$u_{1_{0}}[\mathrm{v}]$ & 3 & 3 \\
\hline$\gamma_{1}, \gamma_{2}$ & $(0.25,0.35)$ & $(0.7,0.6)$ \\
\hline
\end{tabular}

\section{Preliminaries}

In this section, the preliminaries regarding Laguerre filters are introduced. Laguerre functions are particular classes of orthonormal basis functions [5] and they are well known for their orthonormality, which is going to be used in the design of the discrete-time model predictive controller.Thanks to this property, the application of these networks is mainly used in the area of system identification.

The Z-transforms of the discrete-time Laguerre network are written as follows:

$\Gamma_{n}(z)=\Gamma_{n-1}(z) \frac{z^{-1}-a}{1-a z^{-1}} \quad n=2,3, \ldots, N$

with $\Gamma_{1}(z)=\frac{\sqrt{\left(1-a^{2}\right)}}{1-a z^{-1}}$.

where $N$ is the number of Laguerre functions in the network and $a$ is the pole location of the Laguerre network. The scaling factor $a$ is required to be selected by the user, where $a \in[0,1$ [ for the stability of the network. Note that the choice of the parametera is very important to ensure the convergence of the Laguerre functions.

With the relation (5), the Laguerre network is illustrated in Fig.2

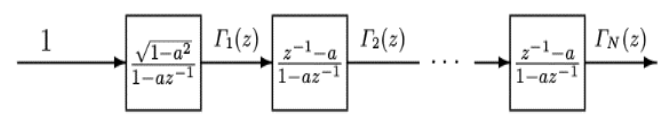

Fig. 2. Illustration of a discrete Laguerre network

The discrete-time Laguerre functions are obtained through the inverse Z-transform of the Laguerre network. The set of Laguerre functions at sample $k$ can be expressed as:

$$
L(k)=\left[\begin{array}{llll}
l_{1}(k) & l_{2}(k) & \ldots & l_{N}(k)
\end{array}\right]^{T}(6)
$$

where $l_{i}(k)$ denotes the inverse Z-transform of $\Gamma_{i}(z, a)$. Taking advantage of the network realization (5), the set of discrete-time Laguerre functions satisfies the following difference equation

$L(k+1)=A_{l} L(k)(7)$

where $A_{l} \in \mathfrak{R}^{N \times N}$ is a function of the parameters $a$ and $\beta=\left(1-a^{2}\right)$

$$
A_{l}=\left[\begin{array}{cccccc}
a & 0 & 0 & 0 & \ldots & \ldots \\
\beta & a & 0 & 0 & \ldots & \ldots \\
-a \beta & \beta & a & 0 & \ldots & \ldots \\
a^{2} \beta & -a \beta & \beta & a & \ldots & \ldots \\
\vdots & \vdots & \vdots & \ddots & \ddots & \ldots \\
(-a)^{N-2} \beta & (-a)^{N-3} \beta & \ldots & \ldots & \beta & a
\end{array}\right]
$$

and the initial condition is given by

$$
L(0)^{T}=\sqrt{\beta}\left[1-a \quad a^{2}-a^{3} \ldots(-a)^{N-1}\right](8)
$$

Referring to [13], it is known that the Laguerre functions become a set of pulse operators when $a=0$ which is used to describe the incremental control trajectory in the traditional MPC approach.

\section{DESCRIPTION OF THE CONTROL SIGNAL TRAJECTORY}

Let recall the description of the predicted control trajectory vector $\Delta U$ that is optimized in the design of a conventional MPC: 
$\Delta U=\left[\begin{array}{lll}\Delta u(k) & \ldots & \Delta u\left(k+N_{c}-1\right)\end{array}\right]^{T}$

At time stepk

$\Delta u(k+j)=\left[\begin{array}{lll}\delta(j) & \ldots & \delta\left(j-N_{c}+1\right)\end{array}\right] \Delta U(10)$

where $N_{c}$ is the control horizon and $\delta$ is the pulse operator. The idea is to use the set of discrete Laguerre functions to approximate the optimal control sequence $[\Delta u(k), \Delta u(k+$ $\left.1), \ldots \ldots ., \Delta u\left(k+N_{c}-1\right)\right]$.

The Laguerre networks are well known for their orthonormality which can be expressed by

$\sum_{k=0}^{\infty} l_{i}(k) l_{j}(k)=\left\{\begin{array}{ll}0 & \text { for } i \neq j \\ 1 & \text { for } i=j\end{array}(11)\right.$

The orthonormal property of the Laguerre functions in (11) will be used in the design of MPC. The key idea in MPC based on Laguerre functions lies in the approximating member of control sequence by a set of Laguerre functions.

At time $k$, the predicted control trajectory vector $\Delta U(k)=[\Delta u(k), \Delta u(k+1), \ldots, \Delta u(k+$ $\left.N_{c}-1\right)$ ] is captured by combining a set of Laguerre functions defined in (6) with a set of Laguerre coefficients $\eta(k)$ in (11) obtained from the online optimization:

$\eta(k)=\left[\begin{array}{llll}c_{1}(k) & c_{2}(k) & \ldots & c_{N}(k)\end{array}\right]^{T}$

Then, all components of the incremental control trajectory at sample $k$ can be approximated by writing as a linear combination as follows:

$$
\left\{\begin{array}{c}
\Delta u(k+l \mid k)=\sum_{n=1}^{N} l_{n}(l) c_{n}(k)=L(l)^{T} \eta(k) \\
\text { for } l=0,1, \ldots, N_{c}-1
\end{array}\right.
$$

where $\Delta u(k \mid k)=u(k \mid k)-u(k-1 \mid k)$

withk being the initial time of the moving horizon window and $l$ being the future sampling. The parameter vector $\eta$ comprises $N$ Laguerre coefficients: $\eta=\left[\begin{array}{llll}c_{1} & c_{2} & \ldots & c_{N}\end{array}\right]^{T}$ and $L(l)^{T}$ is the transposed Laguerre function vector as defined in the difference equation (7).

By using this approximation, the optimization problem (13) can be expressed in terms of coefficient vector $\eta$, instead of $\Delta u(k)$ as in the classic approach.

In the conventional MPC technique, the cost function is

$$
\begin{aligned}
J & =\sum_{l=1}^{N_{p}}\|r(k+l l k)-\hat{y}(k+l l k)\|_{Q}^{2} \\
& +\sum_{l=1}^{N_{c}}\|\Delta u(k+l-1 \mid k)\|_{R}^{2}(14)
\end{aligned}
$$

Therefore, the second term of (14) can be rewritten as:

$$
\begin{aligned}
& \|\Delta u(k+l-1 \mid k)\|_{R}^{2} \\
& =\sum_{\substack{N_{c} \\
N_{p}}}^{N_{p}} \Delta u(k+l-1 \mid k)^{T} R \Delta u(k+l-1 \mid k) \\
& =\sum_{l=1} \Delta u(k+l-1 \mid k)^{T} R \Delta u(k+l-1 \mid k)
\end{aligned}
$$

The last term of (15) is obtained by setting:

$$
\begin{aligned}
\Delta u\left(k+N_{c}\right)=\Delta u\left(k+N_{c}+1\right) & =\cdots \\
& =\Delta u\left(k+N_{p}-1\right)=0
\end{aligned}
$$

Substituting (13) into (15) yields:

$$
\begin{aligned}
& \|\Delta u(k+l-1 \mid k)\|_{R}^{2} \\
& =\sum_{\substack{N_{p} \\
N_{p}}}^{N_{N_{p}}} \Delta u(k+l-1 \mid k)^{T} R \Delta u(k+l-1 \mid k) \\
& =\sum_{l=1} \eta^{T} L(l-1) R L(l-1)^{T} \eta=\eta^{T} R_{L} \eta(17)
\end{aligned}
$$

Where $R_{L} \in \Re^{N \times N}$ is a diagonal matrix with the weighting matrix $R$ on its diagonal and $N_{p}$ is sufficiently large to satisfy the orthonormal property .i.e.,

$\sum_{k=0}^{N_{p}} l_{i}(k) l_{j}(k)=\left\{\begin{array}{ll}0 & \text { for } i \neq j \\ 1 & \text { for } i=j\end{array}(18)\right.$

Thus, the coefficient vector $\eta$ will be optimized and computed in the design. With this design framework, the control horizon $N_{c}$ from the classic MPC approach has vanished. Instead, the number of terms $N\left(N<N_{c}\right)$ is used to describe the complexity of the trajectory in conjunction with the free parameter $a$. 
Furthermore, a long control horizon $N_{c}$ can be achieved without using a large number of parameters, leading to low computational burden and memory storage. In this paper, the MPC based on Laguerre functions is used in the proposed DMPC scheme.

\section{Use of Laguerre functions in MPC design}

Consider the non-augmented state-space model (7) to design the controller is represented as follows:

$$
\begin{gathered}
x(k+1)=A x(k)+B u(k) \\
y(k)=C x(k)
\end{gathered}
$$

withu(k)= $\Delta u(k)+u(k-1)$

At step time $k$, the future state prediction over the horizon $N_{p}$ is given by:

$\hat{x}(k+l \mid k)=A^{l} \hat{x}(k \mid k)+\sum_{s=1}^{l} A^{s-1} B u_{i}(k+l-$ $s \mid k)(20)$

According to the expression in (13), the incremental control trajectory in prediction horizon of sample $k$ can be calculated as: $\Delta u(k+l-1 \mid k)=L^{T}(l-1) \eta(k)$ for $l=$ $1,2, \ldots, N_{p}$ in Laguerre formulation.

Then, the prediction of the future state variables at time $l$ becomes

$$
\begin{array}{r}
\hat{x}(k+l \mid k)=A^{l} \hat{x}(k \mid k)+\sum_{s=1}^{l} A^{s-1} B u(k-1) \\
+\sum_{s=1}^{l}\left[\sum_{h=s}^{l} A^{l-h} B\right] L(s \\
-1)^{T} \eta \\
\text { for } l=1,2, \ldots, N_{p}(21)
\end{array}
$$

And the prediction of the plant output will be

$$
\hat{y}(k+l \mid k)=C A^{l} \hat{x}(k \mid k)+C \sum_{s=1}^{l} A^{s-1} B u(k-1)
$$$$
+C \sum_{s=1}^{l} \quad\left[\sum_{h=s}^{l} A^{l-h} B\right] L(s-1)^{T} \eta
$$

To compute the prediction, the convolution sum

$S_{c}(l)=\sum_{s=1}^{l} \quad\left[\sum_{h=s}^{l} A^{l-h} B\right] L(s-1)^{T}$

Needs to be computed. To this end, note that

$$
\begin{gathered}
S_{c}(1)=B L(0)^{T} \\
S_{c}(2)=[A B+B] L(0)^{T}+B L(1)^{T} \\
=[A+I] B L(0)^{T}+B L(0)^{T} \mathcal{A}_{l}^{T} \\
=S_{c}(1)+A S_{c}(1)+S_{c}(1) \mathcal{A}_{l}^{T}
\end{gathered}
$$

$$
\begin{array}{r}
S_{c}(3)=S_{c}(2)+A^{2} S_{c}(1)+A S_{c}(1) \mathcal{A}_{l}^{T} \\
+S_{c}(1)\left(\mathcal{A}_{l}^{2}\right)^{T} \\
S_{c}(4)=S_{c}(3)+A^{3} S_{c}(1)+A^{2} S_{c}(1) \mathcal{A}_{l}^{T}+ \\
A S_{c}(1)\left(\mathcal{A}_{l}^{2}\right)^{T}+S_{c}(1)\left(\mathcal{A}_{l}^{3}\right)^{T}(24)
\end{array}
$$

Continuing the recursion in (24) reveals that for $l=2,3, \ldots, N_{p}$

$S_{c}(l)=S_{c}(l-1)+\sum_{h=1}^{l} A^{h-1} S_{c}(1)\left(\mathcal{A}_{l}^{(l-h)}\right)^{T}$

with $\quad S_{c}(1)=B L(0)^{T} n$

where the difference equation $L(k+1)=$ $A_{l} L(k)$ is used to generate the set of Laguerre functions.

For a compact form, (22) can be written as

$$
\begin{aligned}
& \begin{array}{c}
\hat{y}(k+l \mid k)=\mathcal{H}(l) \hat{x}(k \mid k)+\Psi(l) u(k-1)+ \\
\phi(l)^{T} \eta
\end{array} \\
& \text { with } \begin{array}{c}
\mathcal{H}(l)=C A^{l} \\
\\
\end{array}(l)=\sum_{s=1}^{l} C A^{s-1} B \\
& \phi(l)^{\mathrm{T}}=C \sum_{s=1}^{l}\left[\sum_{h=s}^{l} A^{l-h} B\right] L(\mathrm{~s}-1)^{\mathrm{T}}
\end{aligned}
$$

\section{Modified cost function}

Since the discrete Laguerre functions are orthonormal for a sufficiently large prediction horizon $N_{p}$, the cost function (14) is equivalent to the modified cost function defined as:

$J=\sum_{l=1}^{N_{p}}\|r(k+l \mid k)-\hat{y}(k+l \mid k)\|_{Q}^{2}+\eta^{T} R_{L} \eta(28)$

The main objective is to find the best control law that will drive the predicted plant output as close as possible to the reference trajectory, moreover this cost function here is a trade-off between error tracking and energy consumption.

\section{E. Unconstrained solution}

The objective is to find the coefficient vector $\eta$ that minimizes Jwithout constraints, by substituting (26) into (28), the optimal solution of the parameter vector $\hat{\eta}$ is 
$\hat{\eta}=$

$\left(\sum_{l=1}^{N_{p}} \phi(l) Q \phi(l)^{T}+R_{L}\right)^{-1} \times$

$\left(\sum_{l=1}^{N_{p}} \phi(l) Q(r(k+l \mid k)-\mathcal{H}(l) \hat{x}(k \mid k)-\right.$

$\Psi(l) u(k-1)))$

Upon obtaining the optimal parameter vector $\hat{\eta}$, the control increment at time step $k$ is as follows:

$\Delta u(k)=\left[\begin{array}{cccc}L_{1}(0)^{T} & 0_{r}^{T} & \ldots & 0_{r}^{T} \\ 0_{r}^{T} & L_{2}(0)^{T} & \ldots & 0_{r}^{T} \\ \vdots & \vdots & \ddots & \vdots \\ 0_{r}^{T} & 0_{r}^{T} & \ldots & L_{p}(0)^{T}\end{array}\right] \hat{\eta}$

where $0_{r}^{T}$, represents a zero block row vector with identical dimension of $L_{r}(0)^{T}$.

Consequently, the control signal $u(k)$ can be calculated as:

$u(k)=u(k-1)+\Delta u(k)$

\section{F. Constrained solution}

One of the interesting features of model predictive control is that the hard constraints on the difference in control variable $\Delta u(k)$, the amplitude of the control signal $u(k)$ and the output variables $y(k)$ are easily implemented through optimization by using quadratic programming.

1) Constraints on the difference of the control variable

Let us assume that the limits on $\Delta U$ are $\Delta u_{\min }$ and $\Delta u_{\max }$ from (13) and at future instant $l$ the constraint is given as:

$\Delta u^{\min } \leq\left[\begin{array}{cccc}L_{1}(l)^{T} & 0_{r} & \ldots & 0_{r} \\ 0_{r} & L_{2}(l)^{T} & \ldots & 0_{r} \\ \vdots & \vdots & \ddots & \vdots \\ 0_{r} & 0_{r} & \ldots & L_{p}(l)^{T}\end{array}\right] \eta \leq \Delta u^{\max }$

\section{2) Constraints on the control input}

Let us suppose that the control input $u(k)$ is bounded by $u_{\min }$ and $u_{\max }$ and the increment of the control action is $u(k)=\sum_{i=0}^{k} \Delta u(k)$, then the inequality constraint is expressed as:

$u^{\min } \leq \Xi(k)+u(k-1) \leq u^{\max }(33)$

where

$$
\Xi(k)=\left[\begin{array}{cccc}
\sum_{i=0}^{k-1} L_{1}(i)^{T} & 0_{r} & \ldots & 0_{r} \\
0_{r} & \sum_{i=0}^{k-1} L_{2}(i)^{T} & \ldots & 0_{r} \\
\vdots & \vdots & \ddots & \vdots \\
0_{r} & 0_{r} & \ldots & \sum_{i=0}^{k-1} L_{p}(i)^{T}
\end{array}\right] \hat{\eta}
$$

\section{3) Constraints on the output variables}

By using (26) and $y_{\min } \leq y(k) \leq y_{\max }$, for the future time instant $l$ the inequality constraint is written as:

$$
\begin{gathered}
y_{\min } \leq \mathcal{H}(l) \hat{x}(k \mid k)+\Psi(l) u(k-1)+\phi(l)^{T} \eta \\
\leq y_{\max }
\end{gathered}
$$

\section{SIMULATION AND RESULTS}

The main advantages of using MPC are constraints handling and Multi-Input MultiOutput support which makes the control of the QTP a challenging problem. In this section the dynamical model of the Quadruple Tank is implemented in Matlab and the system performances are observed via simulations. The objective is to control, independently, the level of the lower tanks without violating the constraints in (36).

The plant described in (4) is discretized with $T_{s}=0.1 \mathrm{~s}$ and initially starts with operating point parameters are given in Table. 2

$$
\begin{aligned}
0 & \leq h_{i} \leq 19.8[\mathrm{Cm}] \\
0 \leq u_{i} \leq 30[\mathrm{~V}](36) &
\end{aligned}
$$

To investigate the performance of the Laguerre MPC in presence of constraints, the simulation results are presented in the next figures, by choosing $N_{p}=30, a=0.5, N=3$ we can see that the controller tracks perfectly the reference set-point with small deviations from the reference when the set-point is changed as a result of interaction of tanks as shown in Fig.3. 

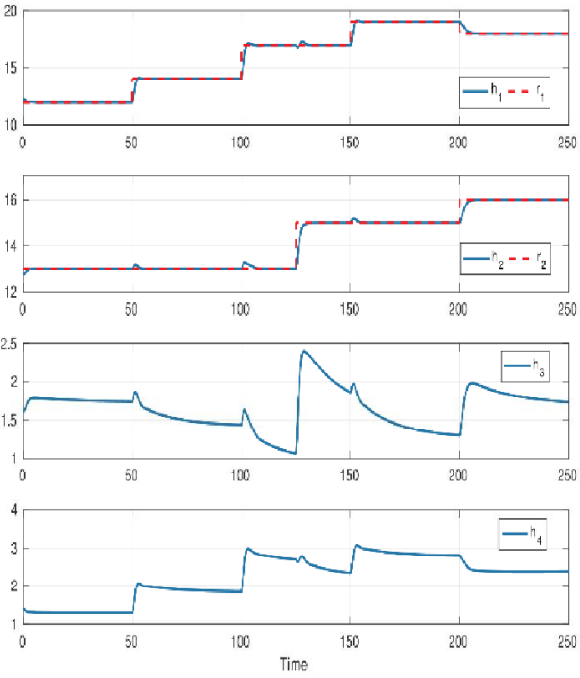

Fig. 3. Closed-loop response with Laguerre MPC.

Fig.4 shows the control inputs (voltages) of the pumps.
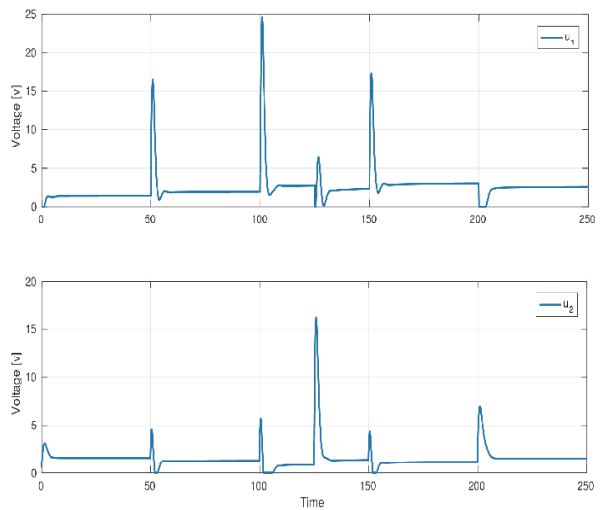

Fig. 4. Control inputs; $\mathrm{N}=3$.

Second scenario is done where the number of Laguerre functions is higher than before $N=$ 5 , from Fig.5 one can see that the output of tank 1 has a slight overshoot due to the increasing of $N$. Moreover, to illustrate the constraints handling by the controller we specified the set-point closer to the limit in the tank 1and changed the reference in tank 2 which cause a small pick in the $h_{1}$ due to the interactions, as expected, the water level didn't exceed the preset value of $y_{\max }$ as seen on the zoom-in given in the first subplot of Fig.5.

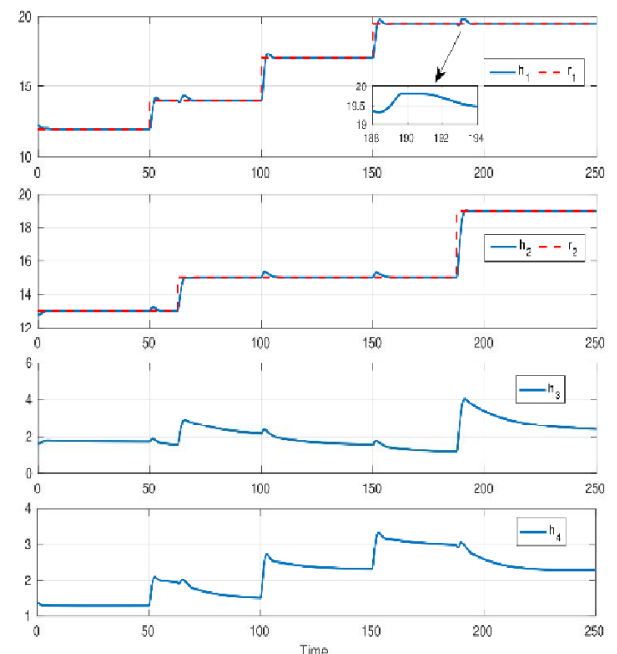

Fig. 5. Closed-loop response with constraints.

Another observation regarding the control action where Fig. 6 shows that the controller respects very well the limits of the pumps voltages defined in (36).
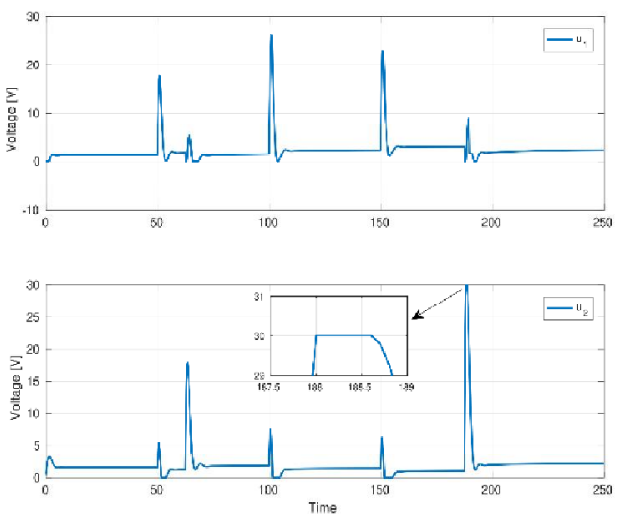

Fig. 6. Control inputs with constraints; $N=5$.

5. CONCLUSION AND FUTURE WORK In this paper, the MPC solution, was proposed for improving the global performance of closed- loop system; it is based on Laguerre functions used in MPC formulation. The proposed methodology is demonstrated on QTPfor a set-point tracking. In case of high process dynamics the satisfactory approximation of $\Delta U$ need a large number of samples. By using Laguerre functions into MPC technique, a long control horizon can be realized without using large number of parameters. Simulation results show that the proposed controller is better than the conventional MPC which need a higher number parameters to achieve a similar response. The extension of the proposed 
controller to nonlinear systems in presence of disturbances will be treated in future work.

\section{References}

[1] D. W. Clarke, C. Mohtadi, and P. Tuffs. Generalized predictive controlpart i. the basic algorithm. Automatica, vol.23, pp.137-148, 1987.

[2] C. E. Garcia and M. Morari. Internal model control. 2. design procedure for multivariable systems. Industrial \& Engineering Chemistry Process Design and Development, vol.24, pp.472-484, 1985.

[3] K. H. Johansson, A. Horch, O. Wijk, and A. Hansson. Teaching multivariable control using the quadruple-tank process. In Proceedings of the 38th IEEE Conference on Decision and Control, vol.1, pp. 807-812. IEEE, 1999.

[4] M. Katebi and M. Moradi. Predictive PID controllers. IEE ProceedingsControl Theory and Applications, vol.148, pp.478-487, 2001.

[5] Y. W. Lee. Statistical theory of communication. American Journal of Physics, vol.29, pp.276278, 1961.

[6] L Wang. Discrete time model predictive control design using laguerre functions. In Proceedings of the 2001 American Control Conference., vol.3, pp.2430-2435, June 2001.

[7] K. Menighed. Commandes cooperatives embarquées et tolérantes aux defauts. PhD thesis, Universite Henri Poincaré-Nancy I, 2010.

[8] M. Morari. Model predictive control: multivariable control technique of choice in the 1990s, 1993.

[9] H. Panagopoulos, K. Astrom, and T. Hagglund. Design of PID controllers based on constrained optimisation. IEE ProceedingsControl Theory and Applications, vol.149, pp.32-40, 2002.

[10] S. J. Shiu and S.. H. Hwang. Sequential design method for multivariable decoupling and multiloop PID controllers. Industrial \& engineering chemistry research, vol.37, pp.107-119, 1998.

[11] G. Stein. LQG-based multivariable design: Frequency domain interpretation. AGARD Lecture Series, 117, 1981.

[12] B. D. Tyreus and W. L. Luyben. Tuning PI controllers for integrator/dead time processes. Industrial \& Engineering Chemistry Research, vol.31, pp.2625-2628, 1992.

[13] L. Wang. Model Predictive Control System Design and Implementation Using MATLAB. Advances in Industrial Control, Springer, 2009.

[14] Q. G. Wang, B. Zou, T. H. Lee, and Q. Bi. Auto-tuning of multivariable PID controllers from decentralized relay feedback. Automatica, vol.33, pp.319-330, 1997.

[15] T. Yamamoto and S. Shah. Design and experimental evaluation of a multivariable selftuning PID controller. IEE Proceedings-Control Theory and Applications, vol.151, pp.645-652, 2004.

[16] R. Yusof, S. Omatu, and M. Khalid. Self-tuning PID control: a multivariable derivation and application. Automatica, vol.30, pp.1975-1981, 1994.

[17] Fayçal CHABNI, Rachid TALEB*, Abderrahmen BENBOUALI, Mohammed Amin BOUTHIBA, "Digital Control Fuzzy Logic for a Water Tank Level Using Arduino", Algerian Journal of Signals and Systems, Vol.1, Issue1, June 2016. pp. 2-10.

[18] Riad BENDIB, Noual BATOUT, Abdelmajid KHELASSI, "Design of Effective Control Schemes for Binary Distillation Columns", Algerian Journal of Signals and Systems, Vol.2, Issue 4, December 2017. pp. 248-254 